\title{
Analysis of Heat Balance of MH Separator
}

\author{
Lukáš Tóth ${ }^{1, *}$, Tomáš Brestovič ${ }^{1}$, Natália Jasminská ${ }^{1}$, and Marián Lázár ${ }^{1}$ \\ ${ }^{1}$ Department of Power Engineering, Faculty of Mechanical Engineering, Technical University of \\ Košice, Slovakia
}

\begin{abstract}
The paper deals with the thermal balance of a metal hydride separator, primarily a tank containing a metal hydride alloy subjected to heating and cooling processes caused by absorption and desorption. As during the equipment operation it is necessary to ensure heating and cooling, the amount of the required heat to be supplied and removed must be identified. The article also deals with hydrogen separation from undesired components of synthetic gas which is formed in a plasma reactor during the waste gasification process.
\end{abstract}

\section{Introduction}

Gradual development of energy extraction from unrecyclable waste has lead to the current development of extraction of syngas with high hydrogen content thanks to the process of high-temperature waste gasification. During this process, higher molecules decompose into basic components from which it is possible to extract hydrogen while applying various syngas treatment methods. As a result of the gradual development of metal hydride alloys, these two technological processes currently overlap and the interest in using metal hydride materials for the extraction of highly pure hydrogen is increasing.

\section{Syngas production using a plasma reactor}

The technology of plasma gasification of waste is based on creating a plasma arc discharge from a flow of inert gas (argon, nitrogen etc.) or from an oxygen-enriched gas. Such plasma-forming gas is transformed, using a high-intensity electric field, into the plasma state. High temperatures along the edges of the plasma arc discharge ensure a constant heat flow which is required for the thermal degradation of waste.

Due to the effects of high bond-dissociation energy, the organic component of waste decomposes into simple gas molecules. The inorganic components, in form of liquid slag and a metal alloy, gather in the lower part of the reactor and are periodically discharged through the tap hole.

Following the cooling and cleaning stages, syngas may be used for energy purposes through the combustion in combustion aggregates, depending on the percentage of the contained combustible components, including mainly hydrogen and carbon monoxide [1].

\footnotetext{
*Corresponding author: lukas.toth@tuke.sk
} 


\subsection{Plasma gasification principle}

The input material for the plasma gasification is waste, including biological waste, communal waste, sledge from waste water treatments, hazardous waste from hospitals and healthcare facilities or certain types of production waste. The first necessary step is the technological treatment of the waste. The waste intended for further processing must meet certain requirements for technological parameters for individual reactor types.

After the waste is processed into sizes more convenient for the handling purposes, it is charged, using a special charging device, into a plasma reactor where subsequently gasification and vitrification processes run. In the reactor, there is a plasma torch which may consist of different types of electrodes. The electrode types are described in Table 1.

The current is supplied to the electrodes and a plasma arc is formed between them, or between the electrode and the reactor bottom. Then the plasma gas is let in the plasma arc. The most frequently used plasma-forming gas is nitrogen, due to its good accessibility, and in the presence of a high-intensity electric field it transforms into plasma. The temperature near the arc ranges from 2.000 to $10.000{ }^{\circ} \mathrm{C}$. In the plasma arch, organic and partially also inorganic materials decompose into simple gaseous and liquid compounds.

Table 1. Parameters of plasma torches with a transferred electric arc.

\begin{tabular}{|c|c|c|c|c|}
\hline Parameter & $\begin{array}{c}\text { Cold } \\
\text { electrode }\end{array}$ & $\begin{array}{c}\text { Hot graphite } \\
\text { electrode }\end{array}$ & $\begin{array}{c}\text { A pair of cold } \\
\text { electrodes }\end{array}$ & $\begin{array}{c}\text { A pair of hot } \\
\text { graphite } \\
\text { electrodes }\end{array}$ \\
\hline Maximum current intensity (A) & 8.000 & $>30.000$ & 5.000 & $>30.000$ \\
\hline Energy efficiency $(\%)$ & $75-80$ & $>85$ & $75-80$ & $>85$ \\
\hline Maximum temperature $\left({ }^{\circ} \mathrm{C}\right)$ & $>3.000$ & $>3.000$ & $>3.000$ & $>3.000$ \\
\hline Use of oxidising atmosphere & Yes & Limited & Yes & Limited \\
\hline Reactor leak-tightness & Good & Good & Good & Good \\
\hline Possibility of automation & Moderate & Good & Moderate & Good \\
\hline Torch service life (hours) & -1.000 & $\begin{array}{c}\text { Electrode is } \\
\text { continuously } \\
\text { supplemented }\end{array}$ & $\sim 1.000$ & $\begin{array}{c}\text { Electrode is } \\
\text { continuously } \\
\text { supplemented }\end{array}$ \\
\hline
\end{tabular}

The resulting products are a vitrificate and syngas with significantly high contents of combustible components, such as $\mathrm{H}_{2}, \mathrm{CO}, \mathrm{CO}_{2}$ a $\mathrm{CH}_{4}$. In the case of high-temperature gasification of communal waste, their content represents more than $90 \%$. In some cases, hydrogen content amounts to as much as $55 \%$ and this fact gave rise to the efforts aiming at efficient hydrogen separation. In the development of new methods of hydrogen separation, greater emphasis is now being put on using metal hydride alloys.

\section{Process of hydrogen storage in metal hydride alloys}

Figures Reactions between hydrogen and metals represent a special type of heterogeneous reactions on the gas/metal phase boundary. The kinetics of hydride formation, with regard to their specific properties, is different from those of other gas/solid reactions. Specific properties of $\mathrm{MH}$ systems include extremely high diffusivity of hydrogen dissolved in a lattice of a metal or an alloy. The process of metal hydration is accompanied by hydrogen diffusion into a lattice of a metal or an alloy (Figure 1) [2]. 


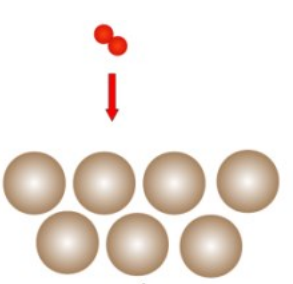

a)

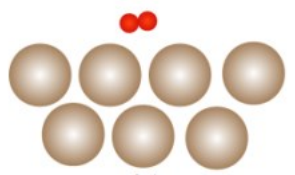

b)



c)



d)

a) $\mathrm{H}_{2}$ molecule approaching the metal surface; b) $\mathrm{H}_{2}$ molecule interaction facilitated by Van der Waals forces (physisorption); c) Hydrogen chemisorption after molecule dissociation; d) Occupation of sub-surface locations and diffusion into the lattice volume

Fig. 1. The interaction between hydrogen and metal.

In the first step of the interaction between hydrogen and metal, a hydrogen molecule approaches the metal surface due to attractive Van der Waals forces; this causes physisorption. The physisorption energy represents $E_{\text {phys }}=6 \mathrm{~kJ} \cdot \mathrm{mol}^{-1}$ of $\mathrm{H}_{2}$. In this process, the molecule of gaseous hydrogen interacts with several atoms on the surface of a solid material.

In the following step of the hydrogen/metal interaction, the hydrogen molecule must overcome the activation barrier in order to form a hydrogen-metal bond and dissociate. This process is referred to as chemisorption. The chemisorption energy $E_{\text {chem }}$ ranges from 20 to $150 \mathrm{~kJ} \cdot \mathrm{mol}^{-1}$ of $\mathrm{H}_{2}$, and thus it is significantly higher than the physisorption energy. Hydrogen molecule dissociation leads to chemisorption and the subsurface positions are occupied by hydrogen atoms. After the hydrogen molecule dissociates on the surface of the metal, hydrogen atoms diffuse into the volume with the resulting formation of MH solid solution, generally referred to as the $\beta$-phase. The kinetics of the process of hydrogen absorption and desorption into intermetallic alloys is affected by several factors, including heat and mass transfer, alloy purity, sizes and shapes of particles etc.

\subsection{Thermodynamic analysis of hydrogen storage in intermetallic hydrides}

Depending on the type and strength of the hydrogen-metal bond $(\mathrm{MH})$, metals may be exothermic absorbers; that is why almost all metal hydride systems are exothermic. Multiple metals and alloys react reversibly with hydrogen with the reaction product being a metal hydride, as described in reaction [8]:

$$
\mathrm{M}_{(\mathrm{s})}+\frac{\mathrm{x}}{2} \mathrm{H}_{2(\mathrm{~g})}^{\stackrel{\text { absorpcia }}{\rightarrow}} \mathrm{MH}_{\mathrm{x}(\mathrm{s})}+Q
$$

wherein $M$ is a metal solid solution or an intermetallic compound), $H$ - hydrogen, $x$ - molar fraction of hydrogen (1), MH - relevant metal hydride, $Q$ - reaction heat $\left(\mathrm{J} \cdot \mathrm{mol}^{-1}\right),(\mathrm{g})$ - gas phase and (s) - solid phase.

With regard to the fact that the hydride entropy is lower than that of a metal and a gaseous hydrogen, the reaction of hydrogen absorption into a metal or an alloy is exothermic, and a reverse reaction of hydrogen desorption from hydride is endothermic.

Therefore, hydrogen absorption is accompanies by thermal energy generation in the $\mathrm{MH}$ system, and during desorption it is necessary to supply thermal energy into the $\mathrm{MH}$ system in order to keep the process running.

Metal and hydrogen form two different types of hydride; the $\alpha$-phase, when only a part of hydrogen is absorbed; and the $\beta$-phase, when hydrogen is fully saturated with metal [3]. 
In the equilibrium state, the chemical potentials of hydrogen in the gaseous phase and hydrogen absorbed into a metal are identical:

$$
\frac{1}{2} \mu_{(\mathrm{g})}=\mu_{(\mathrm{s})} \quad\left(\mathrm{J} \cdot \mathrm{mol}^{-1}\right)
$$

As the internal energy of a hydrogen molecule represents $7 / 2 \mathrm{k} \cdot T$, enthalpy and entropy of a hydrogen molecule may be calculated using equations (3) through (5):

$$
\begin{aligned}
& h_{(\mathrm{g})}=\frac{7}{2} \cdot \mathrm{k} \cdot T-E_{\text {dis }} \\
& s_{(s)}=\frac{7}{2} \cdot \mathrm{k}-\mathrm{k} \cdot \ln \frac{p}{p_{0}(T)} \quad\left(\mathrm{J} \cdot \mathrm{K}^{-1}\right) \\
& p_{0}(T)=\frac{8 \cdot(\pi \cdot \mathrm{k} \cdot T)^{\frac{7}{2}} \cdot m_{\mathrm{H}-\mathrm{H} \cdot r_{\mathrm{H}-\mathrm{H}}^{2}}^{\frac{5}{2}}(\mathrm{~Pa})}{h^{5}}
\end{aligned}
$$

wherein $\mathrm{k}$ is the Boltzmann constant $\left(1.380658 \cdot 10^{-23} \mathrm{~J} \cdot \mathrm{K}^{-1}\right.$, or $\left.8.6173303 \cdot 10^{-5} \mathrm{eV} \cdot \mathrm{K}^{-1}\right), T-$ temperature $(\mathrm{K}), E_{\mathrm{dis}}-$ dissociation energy of hydrogen $\left(E_{\mathrm{dis}}=4.52 \mathrm{eV} / \mathrm{H}_{2}\right), m_{\mathrm{H}-\mathrm{H}}-$ mass of a hydrogen molecule $\left(m_{\mathrm{H}-\mathrm{H}}=3.2751 \cdot 10^{-27} \mathrm{~kg}\right), r^{\mathrm{H}-\mathrm{H}}-$ an interatomic distance of two hydrogen atoms in a $\mathrm{H}_{2}$ molecule $\left(r_{\mathrm{H}-\mathrm{H}}=74 \mathrm{pm}\right), h_{(\mathrm{g})}$ - enthalpy of $\mathrm{H}_{2}(\mathrm{~J}), s_{(\mathrm{s})}-$ entropy of $\mathrm{H}_{2}\left(\mathrm{~J} \cdot \mathrm{K}^{-1}\right)$.

By editing equations (3) and (4), the equation for the chemical potential of gaseous hydrogen is obtained:

$$
\mu_{(\mathrm{g})} \quad=T \cdot \mathrm{k} \cdot \ln \frac{p}{p_{0}(T)}-E_{\mathrm{dis}}=T \cdot \mathrm{k} \cdot \ln \frac{p}{\mathrm{p}_{0}}+\mu_{(g)_{0}} \quad\left(\mathrm{~J} \cdot \mathrm{mol}^{-1}\right)
$$

wherein $p_{0}$ is the standard pressure $(101,325 \mathrm{~Pa})$.

In the solid solution ( $\alpha$-phase), the chemical potential is subject to the equation:

$$
\begin{array}{lll}
\mu_{\alpha}=h_{\alpha}-T \cdot s_{\alpha} & & \left(\mathrm{J} \cdot \mathrm{mol}^{-1}\right) \\
s_{\alpha}=s_{\alpha_{\text {conf }}}+s_{\alpha_{\text {vibelec }}} & & \left(\mathrm{J} \cdot \mathrm{mol}^{-1} \cdot \mathrm{K}^{-1}\right)
\end{array}
$$

wherein $h_{\alpha}$ is enthalpy of the $\alpha$-phase $\left(\mathrm{J} \cdot \mathrm{kg}^{-1} \cdot \mathrm{mol}^{-1}\right), s_{\alpha}$ - entropy of the $\alpha$-phase $\left(\mathrm{J} \cdot \mathrm{mol}^{-1} \cdot \mathrm{K}^{-1}\right)$, $s_{\text {avibelec }}$ - entropy caused by vibration of atoms $\left(\mathrm{J} \cdot \mathrm{mol}^{-1} \cdot \mathrm{K}^{-1}\right), s_{\text {aconf }}-$ configuration entropy $\left(\mathrm{J} \cdot \mathrm{mol}^{-1} \cdot \mathrm{K}^{-1}\right)$ from the formed allocations of $N_{\mathrm{H}}$ hydrogen atoms at various interstitial locations of $N_{\text {is }}$ according to the following equation [4]:

$$
s_{\alpha_{\mathrm{konf}}}=\mathrm{k} \cdot \ln \frac{N_{\mathrm{is}} !}{N_{\mathrm{H}} !\left(N_{\mathrm{is}}-N_{\mathrm{H}}\right) !} \quad\left(\mathrm{J} \cdot \mathrm{mol}^{-1} \cdot \mathrm{K}^{-1}\right)
$$

While applying the Stirling's approximation for a small amount of hydrogen atoms per metal atom, we obtained the equation:

$$
s_{\alpha_{\text {konf }}}=\mathrm{k} \cdot \ln \frac{c_{\mathrm{H}}}{n_{\mathrm{is}}-c_{\mathrm{H}}} \quad\left(\mathrm{J} \cdot \mathrm{mol}^{-1} \cdot \mathrm{K}^{-1}\right)
$$

wherein $n_{\text {is }}$ is the number of interstitial sites per metal atom, $c_{\mathrm{H}}-$ number of hydrogen atoms per metal atom.

After editing equations (7) and (10), a formula was derived for the calculation of the chemical potential of hydrogen in the solid solution ( $\alpha$-phase), as follows: 


$$
\mu_{\alpha}=h_{\alpha}-T \cdot\left(s_{\alpha_{\text {vibelek }}}-\mathrm{k} \cdot \ln \frac{c_{\mathrm{H}}}{n_{\mathrm{is}}-c_{\mathrm{H}}}\right) \quad\left(\mathrm{J} \cdot \mathrm{mol}^{-1}\right)
$$

When considering the equilibrium state (2) of hydrogen, the concentration $c_{\mathrm{H}}$ may be calculated using the following equation:

$$
\begin{gathered}
\frac{c_{\mathrm{H}}}{n_{\mathrm{is}}-c_{\mathrm{H}}}=\sqrt{\frac{p}{p_{0}(T)}} \cdot e^{-\frac{\Delta G_{\mathrm{H}}}{\mathrm{k} \cdot T}} \\
\Delta g=h_{\alpha}-T \cdot s_{\alpha_{\text {vibelek }}}+\frac{1}{2} \mu_{(\mathrm{g})_{0}} \quad\left(\mathrm{~J} \cdot \mathrm{mol}^{-1}\right)
\end{gathered}
$$

or

$$
\begin{aligned}
& \frac{c_{\mathrm{H}}}{n_{\mathrm{is}}-c_{\mathrm{H}}}=\sqrt{\frac{p}{p_{0}(T)}} \cdot e^{-\frac{\Delta G}{\mathrm{R}_{\mathrm{M}} \cdot T}} \\
& \Delta G=\Delta H-T \cdot \Delta S
\end{aligned}
$$

wherein $\Delta G$ is the change in Gibbs free energy $(\mathrm{J}), \mu_{(\mathrm{g}) 0}$ - chemical potential of a hydrogen molecule in normal conditions $\left(\mathrm{J} \cdot \mathrm{mol}^{-1}\right)$ a $R_{M}$ - universal gas constant $\left(R_{M}=8.314472\right.$ $\left.\mathrm{J} \cdot \mathrm{mol}^{-1} \cdot \mathrm{K}^{-1}\right)$.

At low concentrations of hydrogen in the solid phase $\alpha$, hydrogen concentration is directly proportional to the square root of hydrogen pressure in the gas phase. This equation is also known as the Sievert's law:

$$
c_{\mathrm{H}}=\frac{1}{\mathrm{~K}_{\mathrm{s}}} \sqrt{p} \quad\left(\mathrm{~mol} \cdot \mathrm{l}^{-1}\right)
$$

wherein $K_{s}$ is a constant dependent on the temperature of gaseous hydrogen (1).

As the pressure increases, the solid phase becomes saturated with gaseous hydrogen and a metal hydride is formed.

The conversion from the saturated solution of $\alpha$-phase into hydride runs at constant pressure $p$, as described in equation:

$$
\mathrm{MH}_{\mathrm{c}_{\alpha}}\left|\alpha+\frac{1}{2}\left(c_{\beta}-c_{\alpha}\right) \cdot \mathrm{H}_{2} \leftrightarrow \mathrm{MH}_{\mathrm{c}_{\beta}}\right|_{\beta}+Q_{\alpha \rightarrow \beta}
$$

wherein $M H_{c \alpha}$ is a metal hydride system at concentration in the $\alpha$-phase, $c_{\alpha}$ - concentration of gaseous hydrogen in the $\alpha$-phase $\left(\mathrm{mol} \cdot \mathrm{L}^{-1}\right), c_{\beta}$ - concentration of gaseous hydrogen in the $\beta$-phase $\left(\mathrm{mol} \cdot \mathrm{L}^{-1}\right), \mathrm{MH}_{\mathrm{c}}$ is a metal hydride system at concentration in the $\beta$-phase, $Q_{\alpha \rightarrow \beta}$ - heat generated during the $\alpha \rightarrow \beta$ phase transition (J).

In the equilibrium state, the chemical potentials of the gas phase, solid solution and hydride phase are identical:

$$
\mu_{\alpha}\left(p, T, c_{\alpha}\right)=\mu_{\beta}\left(p, T, c_{\beta}\right)=\frac{1}{2} \mu_{(\mathrm{g})}(p, T)=\frac{1}{2} \cdot \mathrm{k} \cdot T \cdot \ln \left(\frac{p_{\mathrm{rov}}(T)}{p_{0}}\right)-\frac{1}{2} \mu_{(\mathrm{g})_{0}}
$$

wherein $\mu_{\alpha}$ is the chemical potential of the $\alpha$-phase $\left(\mathrm{J} \cdot \mathrm{mol}^{-1}\right), \mu_{\beta}$ - chemical potential of the $\beta$-phase $\left(\mathrm{J} \cdot \mathrm{mol}^{-1}\right), \mu_{(\mathrm{g})}-$ chemical potential of the gaseous phase $\left(\mathrm{J} \cdot \mathrm{mol}^{-1}\right)$.

\section{Thermal balance of the tank}

With regard to the need for thermal regulation of the metal hydride tank system, it is necessary, already in the designing stage, to know the quantity of heat which the tank will 
generate while being filled with hydrogen. On the other hand, during hydrogen desorption it must be known how much heat must be supplied to the tank to ensure smooth hydrogen desorption.

Table 2. Energy balance of selected alloys.

\begin{tabular}{|c|c|c|c|c|}
\hline Alloy & LaNi5 & LaNi4.8Al $\mathbf{0 . 2}_{\text {.2 }}$ & $\mathbf{M g H}_{\mathbf{2}}$ & $\mathbf{M g}_{\mathbf{2}} \mathbf{N i}$ \\
\hline$\Delta H\left(\mathrm{~kJ} \mathrm{~kg}^{-1}\right)$ & 15.310 & 16.840 & 37.691 & 64.250 \\
\hline$Q(\mathrm{~J})$ & 688.03 & 756.79 & 1693.83 & 2887.39 \\
\hline
\end{tabular}

During absorption of hydrogen in the experimental device shown in Figure 2, $500 \mathrm{~mL}$ of hydrogen were stored and its total weight was $4.494 \cdot 10^{-5} \mathrm{~kg}$. During the storage of the given amount of hydrogen, the amount of heat released from the alloys is shown in Table 2. The given amount of heat must be removed from the system during absorption, and during desorption the heat must be supplied to the system.

With regard to the system of alloy storage in aluminium pressure vessels, the heat capacity of the vessel must also be taken into count. In this case, a part of the generated heat accumulated in the tank, but it was necessary to ensure sufficient cooling to avoid undesired temperature increase.

A measurement stand was constructed for the system of measuring real parameters, as shown in Figure 2 and Figure 3. The stand was used for measuring and analysing real operating parameters of alloys when compared to the values measured in the laboratory. Due to the fact that a real operation is accompanied with regular heating and cooling cycles, alloy contamination and gradual cyclical degradation, the amount of the heat generated by the alloy is likely to change with an increasing number of measurement cycles because the alloy will be able to absorb a lower portion of hydrogen from the gas.

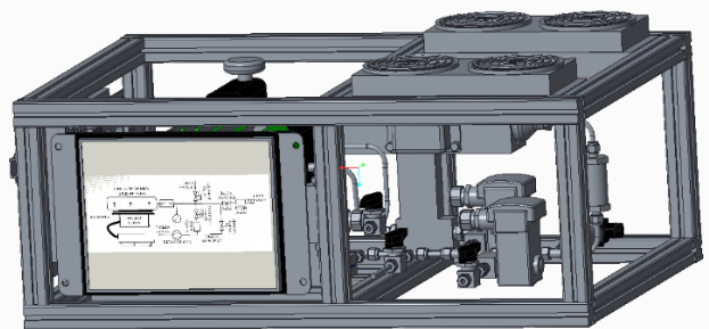

Fig. 2. Alloy testing equipment.

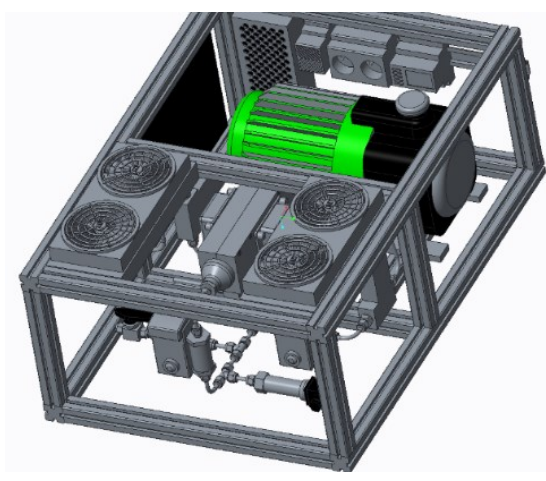

Fig. 3. Alloy testing equipment.

Due to a high percentage of components which might contaminate the metal hydride, it is advisable to increase the percentage of hydrogen in syngas to the highest possible value. This may be carried out while using the physical properties of hydrogen, primarily its density which is significantly lower than that of other syngas components. For the purpose of such physical separation of components, a separator system was created in the modelling software; in the separator system, the gravitation caused the separation of heavier syngas components from hydrogen.

The Ansys CFX simulation was carried out for the purpose of examining the issues regarding the speed of the incoming medium and the locations of the inlet and outlet openings. The most suitable solution, considering the structure dimensions, is the one with 
the inlet opening located on the right bottom side of the equipment and with the opening directed upwards. As shown in Figure 4, such arrangement facilitated slower flowing, and with the optimally set speed of the incoming medium, hydrogen was separated as soon as it entered the equipment. The simulation shows the flow of the incoming gas which then falls down onto the bottom of the equipment due to its significantly higher density.

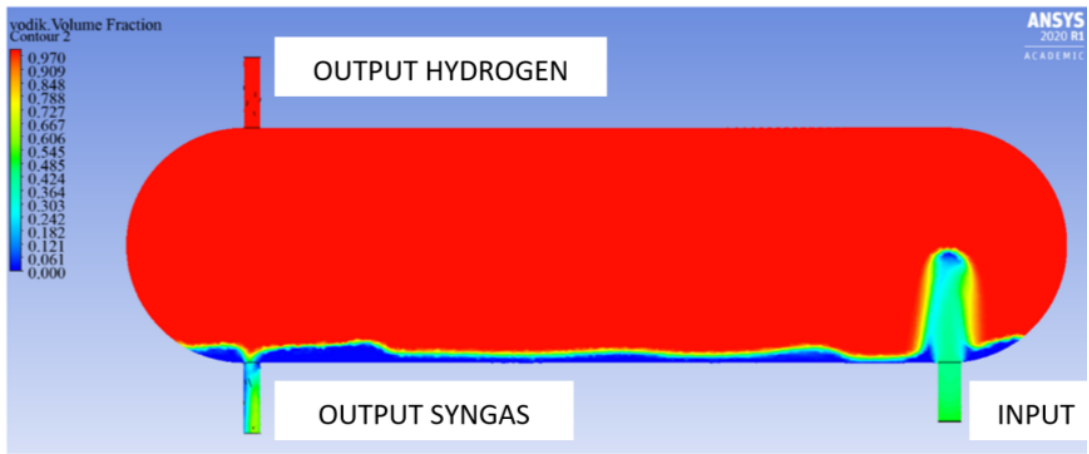

Fig. 4. Hydrogen flow in the separator at the medium speed of $1 \mathrm{~m} \cdot \mathrm{s}^{-1}$ at the inlet.

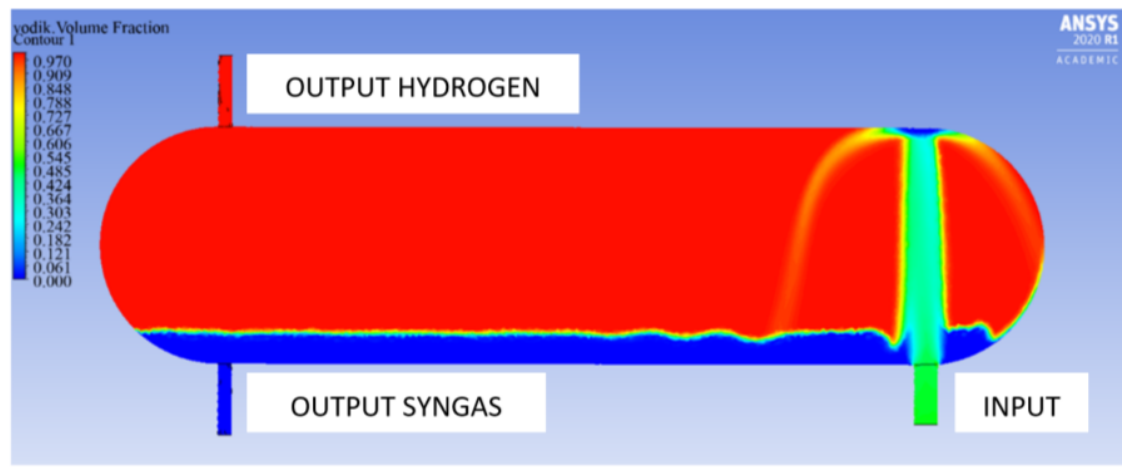

Fig. 5. Hydrogen flow in the separator at the medium speed of $2 \mathrm{~m} \cdot \mathrm{s}^{-1}$ at the outlet.

A factor which is very important in terms of the ability to separate hydrogen from the other syngas components is the size of the outlet piping.

\section{Conclusion}

Within the process of designing a system, its energy efficiency is the key aspect to consider. As it is impossible to identify all types of alloys which will be tested on the equipment, the system with a higher energy demand, based on Peltier thermocouples, was chosen. The performance of thermocouples may be regulated thanks to their modularity and depending on the type of the tested alloy. A potential usable cooling output may be modified by modifying the change in the Peltier thermocouples, from $30 \mathrm{~W}$ to $240 \mathrm{~W}$, while the generated heat capacity ranges from $60 \mathrm{~W}$ to $480 \mathrm{~W}$.

During the series of the measurements of thermal balances of various alloys during the cycle of filling and emptying them, the impact of the repeated filling on the stability of the intermetallic structure will be examined, as well as its ability to absorb the required amount of hydrogen. 
Acknowledgments : This paper was written with the financial support of the granting agency VEGA within the project solution No. 1/0108/19 and No. 1/0626/20 and of the granting agency KEGA within the project solution No. 005TUKE-4/2019.

\section{References}

1. M. Lázár, T. Brestovič, M. Čarnogurská, Vysokoteplotné technológie spracovania odpadu (Košice, 2018)

2. G. Sandrock, S. Suda, L. Schlapbach, Hydrogen in Intermetallic Compounds II: Surface and DynamicProperties, Applications (Springer-Verlag, Berlin, 1992)

3. N. Jasminská, T. Brestovič, M. Lázár, Výroba a uskladnenie vodíka (Košice, 2015)

4. Y. Kaplan, T.N. Veziroglu. Mathematical modelling of hydrogen storage in $\mathrm{LaNi}_{5}$ hydridebed. In: International Journal of Hydrogen Energy. 27, (2003) p. 1027-1038 